\title{
Evaluación de metodologías en el etiquetado de eficiencia energética en edificios
}

M. Laura Canteros, Luis H. Vera, Bruno Natalini (*)

\section{RESUMEN}

El presente trabajo tiene como objetivo el análisis, comparación y evaluación de las metodologías seguidas por las normas actuales sobre el etiquetado de eficiencia energética de edificios entre Argentina y Francia, a fines de establecer convergencias y divergencia. Por un lado, se analizará en Francia, se estudiará el Decreto del 15 de septiembre de 2006: Relativo a la eficiencia energética para los edificios existentes, en venta en Francia metropolitana. Por otro 1ado, en Argentina, la norma IRAM 11900:2017 - Prestaciones energéticas en viviendas. Método de cálculo. A partir del análisis de las normativas argentina y francesa los resultados no son vinculantes dado que difieren en el proceso metodológico.

Palabras clave: Eficiencia energética, IRAM 11900, etiquetado energético.

\section{INTRODUCCIÓN}

La eficiencia energética es la obtención de los mismos bienes y servicios energéticos, con menor consumo energético, con la misma o mayor calidad de vida, con menos contaminación, a un precio inferior al actual, alargando la vida de los recursos y con menos conflicto ${ }^{1}$. La eficiencia energética en los edificios se define en la Directiva para la eficiencia energética de los edificios (Directiva 2002/91/CE del Parlamento Europeo) como la cantidad de energía efectivamente consumida o estimada para responder a las diferentes necesidades ligadas a un uso estandarizado del edificio, lo que puede incluir la calefacción, agua caliente, refrigeración, ventilación e iluminación entre otras.

Según IRAM, la etiqueta de eficiencia energética edilicia tiene sus fundamentos en el ahorro y el consumo responsable de

(*) Facultad de Ingeniería, Universidad Nacional del Nordeste - Mail: malauracanteros@gmail.com

${ }^{1}$ La situación del mundo 1998 : informe anual del Worldwatch Institute sobre medio ambiente y desarrollo (1998) - L R. Brown, C. Flavin y H. F. French - ISBN 84742635149788474263510 
energía eléctrica. Esta etiqueta brinda al usuario la idea del rendimiento que tiene su vivienda, tal y como en los electrodomésticos. Contempla la energía utilizada para calentar, airear, enfriar la envolvente, permitiendo a los habitantes de un recinto alcanzar el confort higrotérmico, a través de sus parámetros constructivos. Las etiquetas de eficiencia energética clasifican, normalmente, los consumos en siete niveles, ordenados de la "A" a la "G", siendo el nivel "A" el más eficiente y el " $G$ " el menos eficiente como se muestra a modo de ejemplo, en la Figura 1.
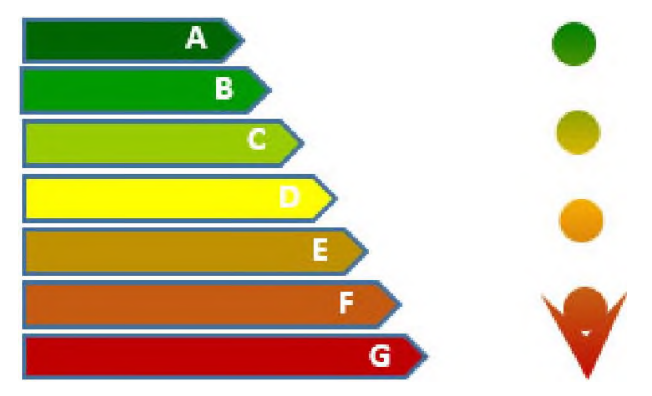

Figura 1- Ejemplo de etiqueta de eficiencia energética. Fuente: elaboración propia

\section{Reglamentación en Francia}

La historia de la reglamentación térmica en edificios en Francia tiene sus comienzos en 1974 con la aparición de la reglamentación térmica edilicia RT 1974 producto de la crisis del petróleo de 1973. Esta crisis ocasionó un aumento de la demanda de petróleo y por consecuente, un aumento en el precio del mismo. Esto provocó que los países industrializados, con una fuerte de- pendencia de la industria petrolera, debieran cambiar sus estrategias para enfrentar la falta del hidrocarburo que ocasionó inestabilidad industrial, económica y una fuerte inflación (Blinder, 1979; Eckstein, 1979; Rupert \& Rapkin, 1985).

Actualmente se encuentra en vigencia la RT 2012 (Collet, 2012). Se prevé su actualización en 2020 con nuevas metas, atendiendo a lo firmado en el Acuerdo de París, Además de la introducción del concepto de "Edificios E+C-“. Su significado yace en el concepto de autoabastecimiento energético de los edificios a través de la implementación de fuentes de energía renovables además de la reducción de emisiones de dióxido de carbono, contemplando la vida útil del edificio (JDN, 2018). Los objetivos de la RT 1974 y sus posteriores modificaciones se representan en la Tabla 1.

En cuanto a las etiquetas de eficiencia energética de edificios, la principal norma europea es la Directiva de eficiencia energética en edificios (EPBD: Energy Performance of Buildings Directive). Esta estableció en 2003 que todos los estados miembros de la UE disponían de 3 años para acordar un certificado de eficiencia energética en los edificios en venta 0 alquiler. Esta directiva de la UE conduce a 1a República Francesa a la necesidad de establecer las bases para la certificación.

Entonces, se establecen métodos según los distintos tipos de edificios para realizar un Diagnóstico de Eficiencia Energética (DPE por sus siglas en francés) siendo válido por 10 años a partir de la fecha de su 


\begin{tabular}{|c|c|}
\hline RT "AÑO"Objetivo & Modificaciones representativas \\
\hline $\begin{array}{l}\text { RT 1974: Reducir en un } 25 \% \text { el } \\
\text { consumo de energia en los edificios. }\end{array}$ & Define el coeficiente $G$ de pérdidas globales de energia. \\
\hline $\begin{array}{c}\text { RT 1982: Aumentar un 20\% la ganancia } \\
\text { de energía. }\end{array}$ & $\begin{array}{l}\text { Define el coeficiente B de "Necesidad de calefacción" teniendo en } \\
\text { cuenta los aportes exteriores e interiores, aportes solares debidos a } \\
\text { la orientación de vidriados. Vuelve obligatoria la aplicación de } \\
\text { estándares sobre las aislaciones. }\end{array}$ \\
\hline $\begin{array}{l}\text { RT 1988: Elección de las tecnologías } \\
\text { menos onerosas para lograr el objetivo } \\
\text { fijado }\end{array}$ & $\begin{array}{l}\text { Define el coeficiente } C \text { que tiene en cuenta el conjunto de } \\
\text { necesidades de calefacción y de agua caliente de uso sanitario, } \\
\text { contemplando el rendimiento de los equipos. Se puede llegar al } \\
\text { objetivo fijado con equipos de buenos rendimientos o reforzando el } \\
\text { aislamiento. Aparecen las consideraciones sobre los equipos de } \\
\text { ventilación y de refrigeración. }\end{array}$ \\
\hline $\begin{array}{c}\text { RT } 2000 \text { : Reducción del } 20 \% \text { de } \\
\text { consumos máximos para viviendas y de } \\
\text { un } 40 \% \text { para edificios terciarios respecto } \\
\text { a las RT } 1988\end{array}$ & $\begin{array}{l}\text { Se tienen en cuenta los parámetros de verano como la apertura de } \\
\text { ventanas y las temperaturas máximas, aparecen las consideraciones } \\
\text { de temperaturas medias en función de la región climática, la inercia } \\
\text { témica y la protección solar. Se considera la "eficiencia" de todos } \\
\text { los conjuntos. }\end{array}$ \\
\hline $\begin{array}{l}\text { RT 2005: Apunta a una reducción del } \\
40 \% \text { del consumo energético entre } 2000 \\
\text { y } 2020 \text {, así como la mejora de un } 15 \% \\
\text { en la eficiencia energética de los } \\
\text { edificios nuevos. }\end{array}$ & $\begin{array}{l}\text { Integra la construcción bioclimática y la evalúa tanto como para la } \\
\text { disminución de calefacción como para el confort de verano. } \\
\text { Introduce las energías renovables en los cáleulos. }\end{array}$ \\
\hline $\begin{array}{l}\text { RT } 2012 \\
\text { (en vigencia): Los nuevos edificios no } \\
\text { deberán consumir más de } 50 \\
\mathrm{kWh} / \mathrm{m}^{2} / \text { año. }\end{array}$ & $\begin{array}{l}\text { Define la Bbio, representa la necesidad bioclimática y evalúa la } \\
\text { eficiencia energética de un edificio y establece un límite máximo } \\
\text { "Bbiomax". Reemplaza la Ubat de la RT 2005. Define el Cep, } \\
\text { consumo de energías primarias. Define la Tic, temperatura interior } \\
\text { convencional, corresponde con un wivel de confort estival en } \\
\text { cuanto al sistema de enfriamiento. }\end{array}$ \\
\hline $\begin{array}{l}\text { RT } 2020 \text { (entrará en vigencia a partir del } \\
2020 \text { ): Edificios de energia positiva. } \\
\text { Edificios pasivos con un } 15 \text { a } 20 \% \text { de } \\
\text { producción de energia sobre una } \\
\text { vivienda convencional. }\end{array}$ & 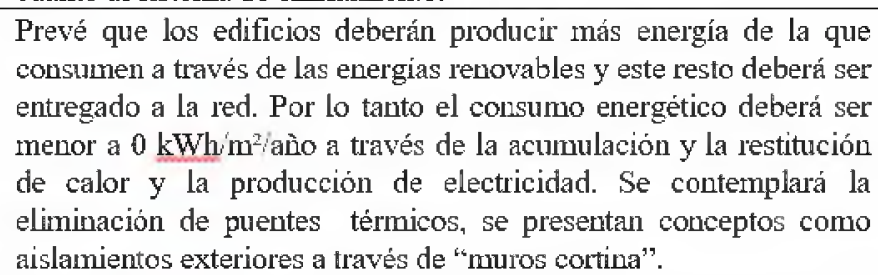 \\
\hline
\end{tabular}

Tabla 1 - Modificaciones de las reglamentaciones térmicas en Francia y los objetivos a sus correspondientes años.

confección. El diagnóstico cobra carácter de cumplimiento obligatorio:

- Para la venta de un inmueble: 1 de septiembre de 2006

- Para el alquiler de un inmueble: $1 \mathrm{ju}$ lio de 2007

- Para obtención de un permiso de construcción de un edificio nuevo: 30 de junio de 2007

- Presentación obligatoria para los edificios públicos: 2 de enero de 2008

A su vez, se establecen una serie de recomendaciones para mejorar el rendimiento edilicio así como también las economías monetarias de la inversión y el tiempo de recupero de los gastos. 


\section{Reglamentación en Argentina}

Argentina es un país en vías de desarrollo, donde actualmente, la División de Planeamiento de la Secretaría de Energía de la Nación ha formulado distintas fases de un Plan Energético de largo plazo integrando distintas metodologías a fin de construir un futuro energético sostenible en el país. Atendiéndose a esta iniciativa, Argentina firmó y ratificó el "Acuerdo de París" el día 21 de septiembre de 2016. Según el Artículo 2 de la Revisión de la Contribución Determinada a Nivel Nacional de Argentina (2016) "La Argentina no excederá la emisión neta de 483 millones de toneladas de dióxido de carbono equivalente ( $\mathrm{tCO} 2$ eq) en el año 2030". Según el Segundo Informe Bienal de Actualización de la República Argentina en la Convención Marco de Las Naciones Unidas sobre el Cambio Climático, 2017, las mitigaciones de CO2 en 2014, alcanzaron un total de 368 millones de toneladas. Las normativas actuales en nuestro país en materia de eficiencia energética atienen a políticas de reducción de emisión de $\mathrm{CO} 2$ tales como las que se contemplan en políticas internas de ambos países, en función de seguir los lineamientos establecidos por protocolos internacionales. Según el Plan de reducción de emisiones de gases de efecto invernadero, presentado en la Conferencia del clima de la ONU (COP22), Argentina pretende reducir en un $18 \%$ sus emisiones de dióxido de carbono para el año 2030 y hasta un $37 \%$ si se recibe ayuda financiera externa.

Considerando lo expuesto, es necesario evaluar la principal fuente de generación de $\mathrm{CO} 2$ y tomando como base estudios que han demostrado que las edificaciones son los principales responsables del consumo de energía y de las emisiones de dióxido de carbono a nivel mundial (Hsu, 2014). El impacto del consumo energético por parte de las viviendas se ha traducido en esfuerzos para mejorar las prestaciones energéticas en viviendas (Lu et al., 2015). Este consumo de energía en el sector residencial y comercial en la región NEA (Nordeste Argentino) alcanzan, según el informe de la ADEERA ${ }^{2}$ de 2018, los valores presentados en la tabla 2 .

\begin{tabular}{|c|c|c|c|c|}
\cline { 2 - 4 } \multicolumn{1}{c|}{} & \multicolumn{4}{|c|}{ PORCENTAJE DE CONSUMO DEL TOTAL } \\
\hline \multirow{2}{*}{ Provincia } & Residencial & \multicolumn{2}{|c|}{ No Residencial } & Grandes Usuarios \\
\cline { 2 - 5 } Formosa & $<10 \mathrm{~kW}$ & $<300 \mathrm{~kW}$ & $\geq 300 \mathrm{~kW}$ & \\
\hline Corrientes & $70,19 \%$ & $22,51 \%$ & $5,22 \%$ & $2,08 \%$ \\
\hline Chaco & $63,21 \%$ & $23,66 \%$ & $6,95 \%$ & $6,19 \%$ \\
\hline Misiones & $70,63 \%$ & $24,53 \%$ & $3,92 \%$ & $0,62 \%$ \\
\hline
\end{tabular}

Tabla 2 - Porcentaje de consumo de energía eléctrica en Formosa, Corrientes, Chaco y Misiones. Fuente: ADEERA

${ }^{1}$ Asociación de Distribuidores de Energía Eléctrica de la República Argentina 


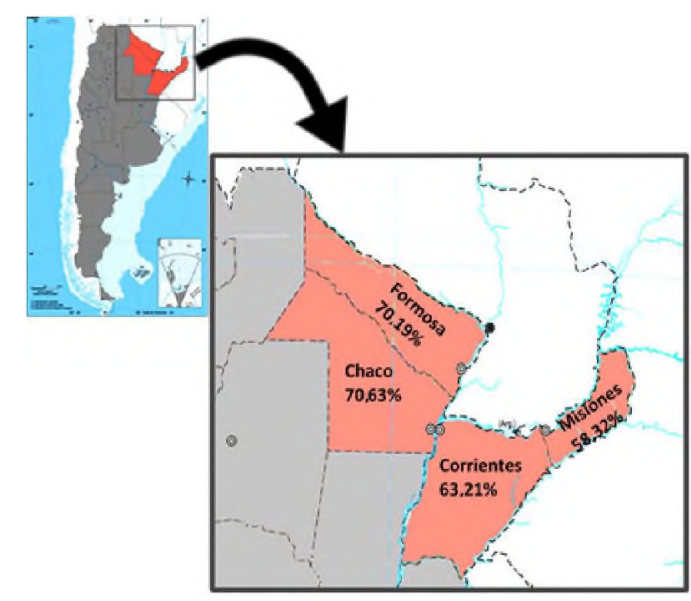

Figura 2

En la Figura 2, se presenta el mapa ampliado donde se muestran los consumos residenciales porcentuales de energía eléctrica en las provincias de Formosa, Corrientes, Chaco y Misiones.

En Argentina, el etiquetado de eficiencia energética de edificios se realiza a través de la norma IRAM 11900. Esta norma tiene dos versiones desde la primera publicada en 2010: IRAM 11900:2010 - Etiqueta de eficiencia energética de calefacción de edificios. Clasificación según la transmitancia térmica de la envolvente. La segunda versión es su modificación y reemplazo en 2017: IRAM 11900:2017 - Prestaciones energéticas en viviendas. Métodos de cálculo.

La norma IRAM 11900 versión 2010 establece el nivel de eficiencia energética a través de un método simplificado, aplicado a las envolventes y superficies susceptibles a ser calefaccionadas a partir de la transmitancia térmica de las envolventes.

En dicho método se utilizó la temperatura media ponderada entre la superficie interior de la envolvente y la temperatura interior de diseño, en grados Celsius, como indicador del nivel de eficiencia energética. Ordenándose los valores y agrupándolos en la etiqueta como muestra la Tabla 3. Es importante destacar que la temperatura no es energía, por lo cual, es un concepto errado vincular eficiencia energética solamente utilizando el parámetro temperatura.

\begin{tabular}{|c|c|}
\hline $\begin{array}{l}\text { Clases de } \\
\text { eficiencia } \\
\text { energética }\end{array}$ & Condición' ${ }^{13}$ \\
\hline A & $\tau_{m} \subseteq 1{ }^{\circ} \mathrm{C}$ \\
\hline B & $1^{\circ} \mathrm{C}<\tau_{m} \leqslant 1,5^{\circ} \mathrm{C}$ \\
\hline $\mathrm{c}$ & $1,5^{\circ} \mathrm{C} \in r_{m} \leq 2^{\circ} \mathrm{C}$ \\
\hline D & $2^{\circ} \mathrm{C}<\tau_{\mathrm{m}} \leqslant 2,5^{\circ} \mathrm{C}$ \\
\hline$E$ & $2,5^{\circ} \mathrm{C}<\tau \leqslant 3^{\circ} \mathrm{C}$ \\
\hline$F$ & $3^{\circ} \mathrm{C}<t_{n} \leq 3.5^{\circ} \mathrm{C}$ \\
\hline $\mathrm{G}$ & $3,5^{\circ} \mathrm{C}<\tau_{n} \leqslant 4^{\circ} \mathrm{C}$ \\
\hline H & $t_{\text {网 }}>4{ }^{\infty} \mathrm{C}$ \\
\hline \multicolumn{2}{|c|}{ 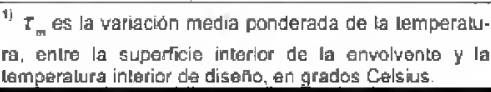 } \\
\hline
\end{tabular}

Tabla 3- Extraida de la norma IRAM - 11900:2010 Tabla 1 pág. 6

\section{METODOLOGÍA}

Para abordar el análisis primeramente, se analizan en profundidad las normativas de eficiencia energética en viviendas unifamiliares vigentes en Francia y en Argentina de forma separada, a fines de buscar puntos en común, así como también convergencias o divergencias entre las mismas.

A continuación, se procede a estudiar una vivienda específica, relevada y etiquetada con la metodología francesa, en la región de Languedoc-Rosellón, en la localidad de Saint-Papoul, perteneciente al 
departamento del Aude, a 33 kilómetros de Carcasona.

Luego, se le aplica la normativa Argentina vigente para la evaluación numérica de los resultados obtenidos y su posterior comparación y conclusión.

El punto de referencia tomado es Francia, dada la vasta trayectoria en reglamentación térmica edilicia que se remite a 1a RT del año 1974.

Actualmente, los esfuerzos de disminución de desprendimientos de $\mathrm{CO} 2$ se ven reflejados en la Directiva 2010/30/EU, donde se exige que se ponga a disposición del propietario o, por parte del propietario, a disposición del posible comprador o inquilino, de un edificio construidos, vendido o alquilado, según corresponda, un certificado de eficiencia energética. Esto se traduce en la necesidad de una metodología efectiva y de una base de datos que ofrezca un nivel de comprensión accesible al común de las personas. Lo cual resulta en una metodología sencilla de abordar e interpretar; adecuada para el presente trabajo.

El resultado de estos lineamientos permitieron que Francia obtenga el segundo mejor puntaje en eficiencia energética a nivel global de acuerdo con el International Energy Efficiency Scorecard 2018 publicado por el Consejo Estadounidense para una Economía Energéticamente Eficiente (ACEEE).

\section{ANÁLISIS DE NORMAS SEGÚN SUS METODOLOGÍAS}

Norma Argentina. IRAM 11900:2017

\section{- Prestaciones energéticas en viviendas. Métodos de cálculo}

El campo de aplicación de la versión actual (2017) se reserva a las prestaciones energéticas para viviendas unifamiliares y/o unidades funcionales de edificios multifamiliares destinadas a uso residencial. En los alcances de dicha versión, se expresa establecer una evaluación cualitativa (según se especifica en la norma) para clasificar el aporte de las medidas de diseño de acondicionamiento de una vivienda.

"Se define a la prestación energética como el uso final de energía convencional que contribuye a la demanda energética de la vivienda mediante los siguientes servicios: la calefacción, la refrigeración, la iluminación artificial de interiores y el calentamiento de agua sanitaria." Las prestaciones energéticas se clasifican, según la norma en:

- Energía necesaria para calefacción y refrigeración

- Energía para A.C.S. (Agua Caliente Sanitaria)

- Energía para la iluminación

- Energía solar térmica y fotovoltaica

Según lo expuesto, el etiquetado que propone la norma IRAM, puede resumirse según lo presentado en la Figura 3, en función de los factores que se consideran para la calificación energética a modo de diagrama de flujo. La norma IRAM conduce a una etiqueta donde los valores de prestaciones energéticas no se correlacionan ni vinculan entre sí, por lo tanto se presentan en forma separada como se muestra en la Figura 4. 


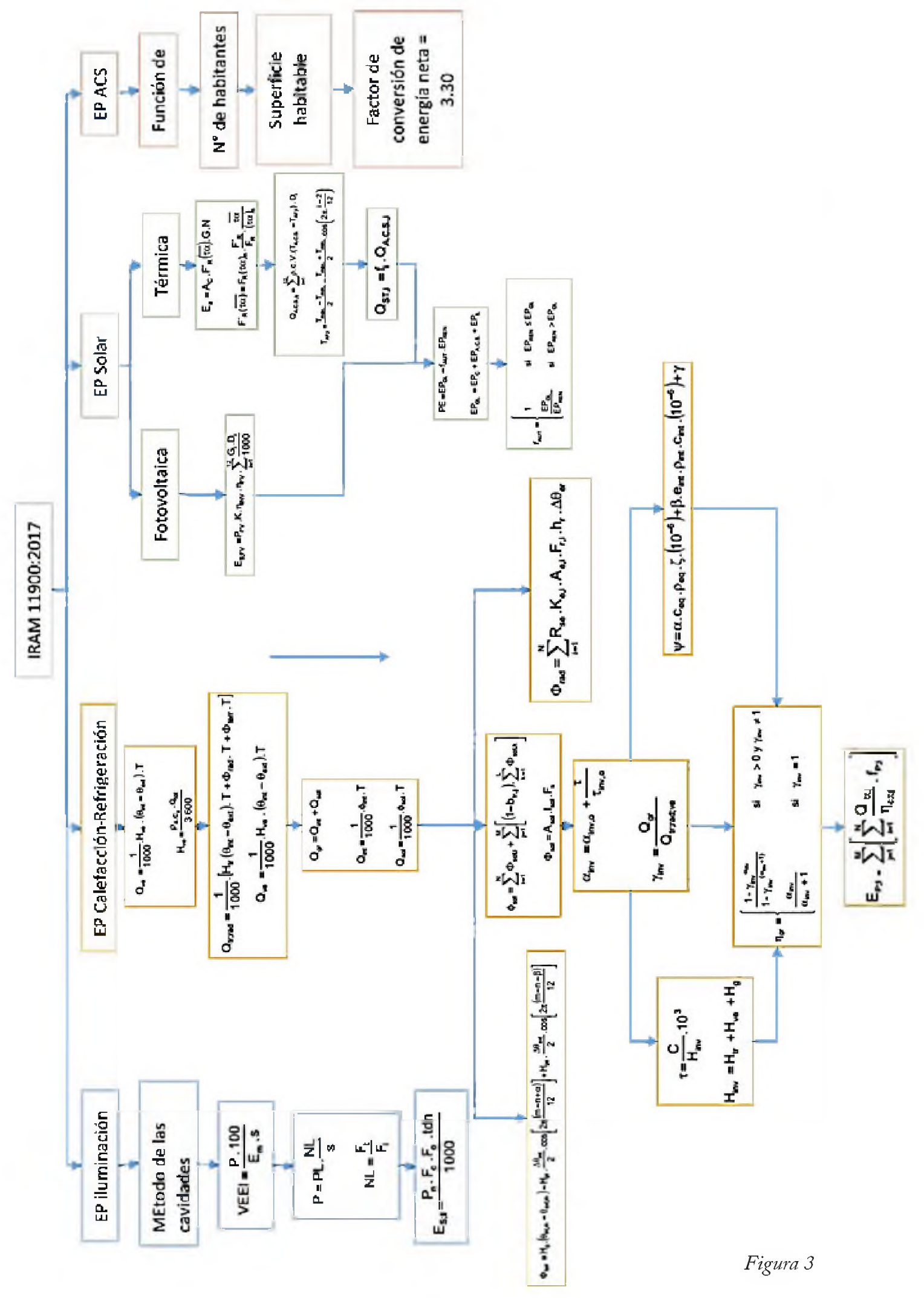




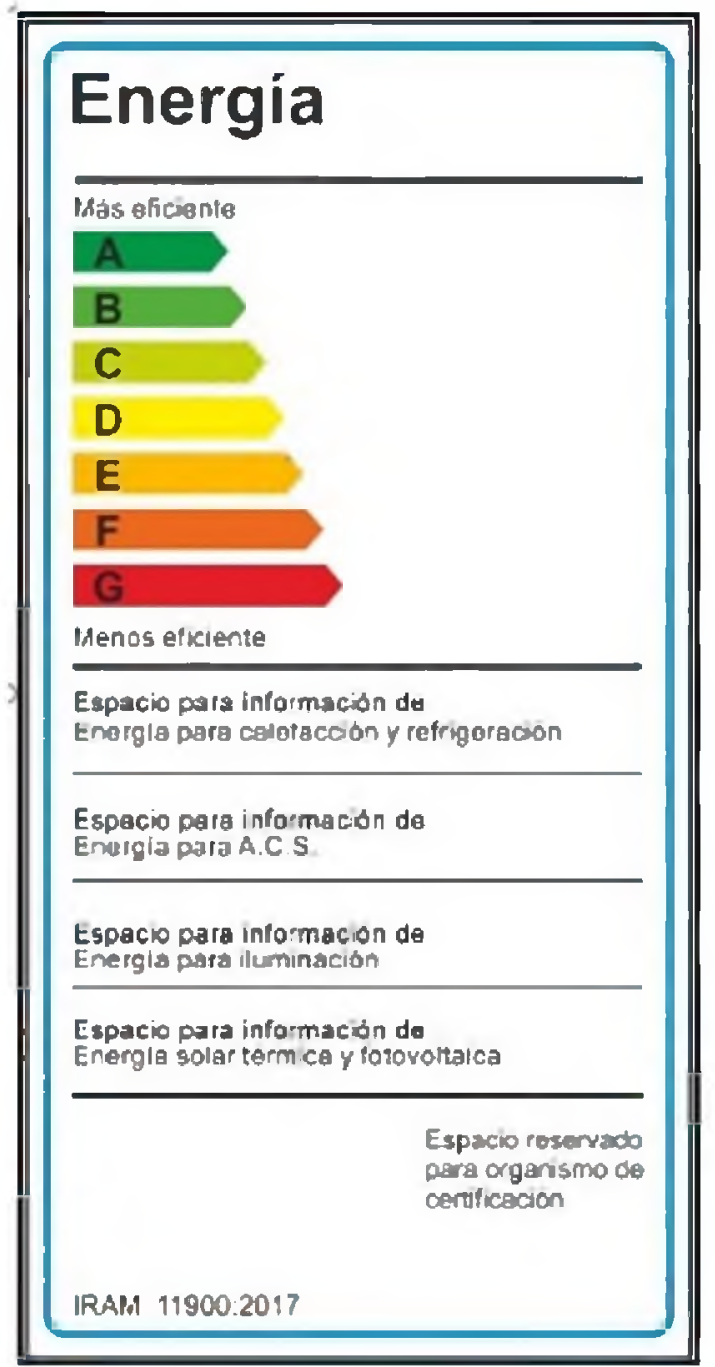

Figura 4 - Ejemplo de etiqueta de la norma IRAM 11900 sin valores

\section{Norma francesa: DPE - Diagnóstico de Prestaciones Energéticas}

E1 DPE informa sobre el rendimiento energético de una vivienda o edificio, a través de la evaluación de del consumo de energía y el impacto del mismo en términos de emisión de gas de efecto invernadero, dentro del cuadro de políticas europeas de energía, establecido para disminuir el consumo de energía de los edificios y limi- tar las emisiones de gases de efecto invernadero (Ministère de la transition écologique et solidaire, 2018). En el DPE deben aparecer:

1- Identificación del edificio;

2- Descripción de las características térmicas y geométricas del edificio, de los equipos de energía instalados en la morada, entre los cuales se contemplan también los equipos que producen o utilizan energías de origen renovable;

3- Cantidad de energía primaria, por año, necesaria para la calefacción de la vivienda, para el calentamiento de agua caliente sanitaria (ACS) así como también para el enfriamiento de las habitaciones;

4- Evaluación en euros sobre el consumo de energías finales por año;

5- Etiqueta de eficiencia energética tipo "ENERGÍA" en kWh/m2año;

6- Etiqueta de eficiencia energética tipo "HUELLA DE CARBONO" en $\mathrm{kgCO} 2 / \mathrm{m}^{2}$ año;

7- Cantidad de energía primaria renovable producida, siempre y cuando esta cantidad pueda ser estimada o medida;

8- Para el caso de calderas presentes en la instalación, último informe de revisión y mantenimiento;

9- Recomendaciones de trabajos y de mejoras térmicas del lote y de los equipos a los efectos de reducir el consumo energético;

10- Fecha de decreto vigente el día de la realización del diagnóstico que fija los precios de las tarifas de energías.

Para establecer los valores numéricos de energías que permiten establecer una 
clasificación y posterior posible comparación con otro inmueble se sigue la metodología presentada a continuación.

1. Cálculo de consumo final de energía anual

a. Metodología convencional

b. Metodología por facturación (no aplica a este caso de estudio)

2. Conversión de energías finales (EF) en energía primaria (EP)

3. Clasificación según etiquetas

a. Etiqueta energética

b. Etiqueta emisiones de $\mathrm{CO} 2$

1. Cálculo de consumo final de energía anual

El decreto del 15 de septiembre de 2006 estima el consumo energético de un edificio, necesario para la calefacción de la vivienda, para el calentamiento de agua caliente sanitaria (ACS) así como también para el enfriamiento de las habitaciones del lote.
Entre los métodos convencionales aprobados por decreto del 6 de mayo de 2008, "Portant confrmation de l'approbation de diverses métbodes de calcul pour le diagnostic de performance énergétique en France métropolitaine" se encuentran, el 3CLDPE, Comfie-DPE y DEL6-DPE. Estos se aplican a viviendas, edificios y departamentos con sistema de calentamiento de agua individual, construidos después de 1948.

El cálculo de las energías consumidas se realiza a través del método de facturación en construidos antes de 1948, en departamentos con sistema colectivo de calefacción de agua y edificios terciarios, construidos tanto antes como después de 1948. Este método toma como consumo final anual de energías al consumo promedio de los últimos tres años que aparece en la factura. De esta forma se ordena como se muestra en la Tabla 4.

\begin{tabular}{|c|c|c|c|}
\hline \multirow{2}{*}{ Tipo de edificación } & \multicolumn{2}{|l|}{ An̆o de construcción } & \multirow{2}{*}{$\begin{array}{l}\text { Tipo de } \\
\text { centificación }\end{array}$} \\
\hline & Antes de 1948 & Después de 1948 & \\
\hline Vivienda umifonilion & DPE método por facturación & DPE método conencional & \multirow{3}{*}{$\begin{array}{l}D P E \\
\text { mencion }\end{array}$} \\
\hline $\begin{array}{l}\text { Departamento con sistena de } \\
\text { calentamiento colectivo }\end{array}$ & DPE método por facturación & DPE método por facturación & \\
\hline $\begin{array}{l}\text { Departamento con sistema de } \\
\text { calentaniento individud }\end{array}$ & DPE método por facturación & DPE método convencional & \\
\hline Edificios tercianios & DPE método por facturación & DPE método por facturación & \multirow{2}{*}{$\begin{array}{l}D P E \\
\text { mención }\end{array}$} \\
\hline Edificios de vivienda colectivos & DPE método por facturación & DPE método comencionat & \\
\hline
\end{tabular}

Tabla 4- Método en función del tipo y año de construcción del inmueble

Dado que en el presente trabajo se analiza una vivienda unifamiliar de construcción posterior a 1948, se aplicará el Método convencional de evaluación de eficiencia energética. a. Metodología convencional de diagnóstico de eficiencia energética para una vivienda en venta: Se explica a continuación el procedimiento para el cálculo de energías finales consumidas por año con el méto- 


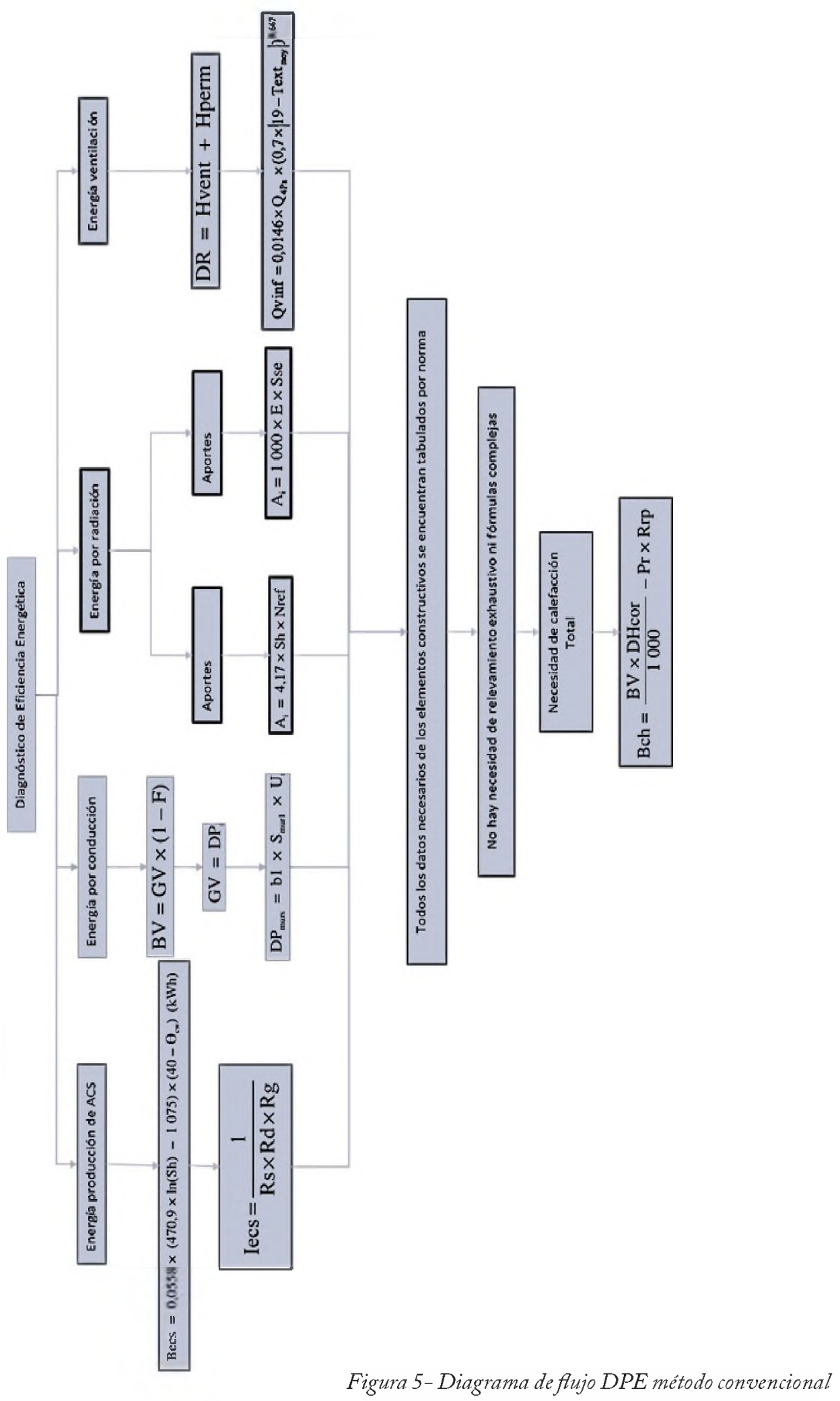


do generalizado por la Agencia Nacional del hábitat (Agence Nationale de l'babitatAnab), el método "3CL" para una vivienda familiar en venta dado que ésta fue concebida especialmente para el cálculo del DPE para edificios de vivienda construidos después de 1948 (ANAH, 2014). Resumiendo, la metodología convencional se puede abordar como se presenta en la Figura 5.

2. Conversión de energías finales (EF) en energías primarias (EP): La cantidad anual de energía primaria en función del tipo de consumo, resultantes de las canti- dades de energía mencionadas precedentemente se expresan a partir de los siguientes factores de conversión: $(+2,58)$ para la electricidad y $(+1)$ para los otros tipos de energías

\section{Clasificación según etiquetas}

a- Etiqueta de eficiencia energética: Para clasificar los resultados se disponen una serie de etiquetas donde se expresa la energía en $\mathrm{kWh} / \mathrm{m}^{2}$ año. Se diferencian en dos tipos, edificios habitacionales (viviendas) y edificios no habitacionales, como se muestra en la Tabla 5 .

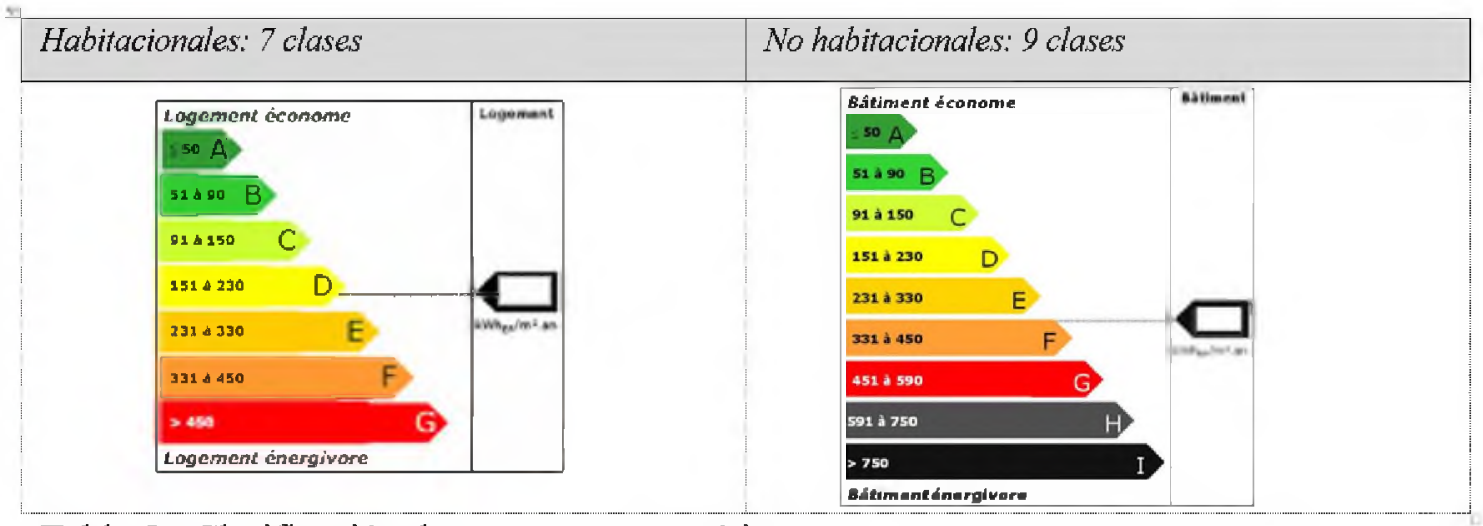

Tabla 5 - Clasificación de consumos energéticos

b- Etiqueta de emisiones de CO2: $\mathrm{Se}$ expresa el consumo de energía en desprendimiento de CO2. E1 método consiste en multiplicar el consumo de energía primaria por un coeficiente de transformación previsto en la derogación del 15 de septiembre de 2006. Los coeficientes serán diferentes si se utiliza el método convencional o el de facturación.

A continuación se clasifican las can- tidades emitidas de $\mathrm{CO} 2$ expresadas en $\mathrm{kgCO} 2 / \mathrm{m}^{2}$ año con la Tabla 6, distinguiéndose en edificios de uso habitacional o no habitacional.

\section{RESULTADOS Y DISCUSIÓN}

De la aplicación de la norma IRAM en la vivienda localizada en Saint-Papoul se obtuvieron los valores listados en la tabla 7 , presentados en la Figura 6. 


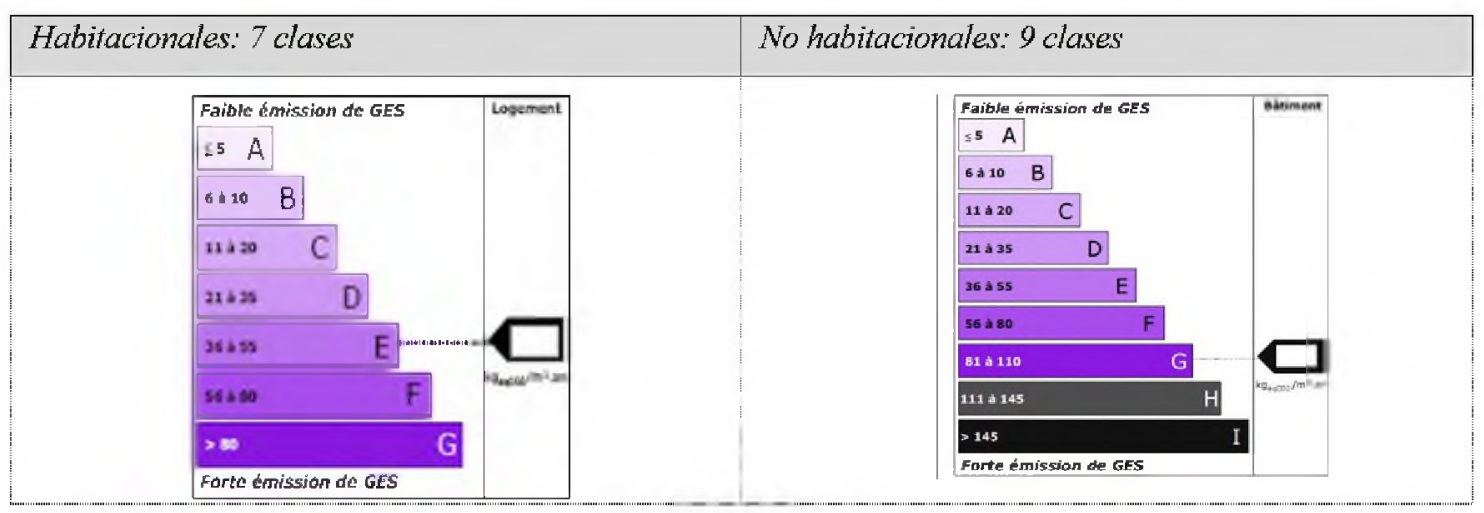

Tabla 6- Clasificación en función de las emisiones de $\mathrm{CO} 2$

\begin{tabular}{|c|c|}
\hline EP Calefacción y Refrigeración & \begin{tabular}{c}
$60,26 \mathrm{kWh} / \mathrm{m}^{2}$ año \\
\hline EP A.C.S.
\end{tabular} \\
\hline EP Iluminación & $25,87 \mathrm{kWh} / \mathrm{m}^{2}$ año \\
\hline EP Solar & $0,23 \mathrm{kWh} / \mathrm{m}^{2}$ año \\
\hline Total & $0 \mathrm{kWh} / \mathrm{m}^{2}$ año \\
\hline$E P$ \\
\hline$E P$
\end{tabular}

Tabla 7- Resultados de aplicación de norma $\mathbb{R A M}$

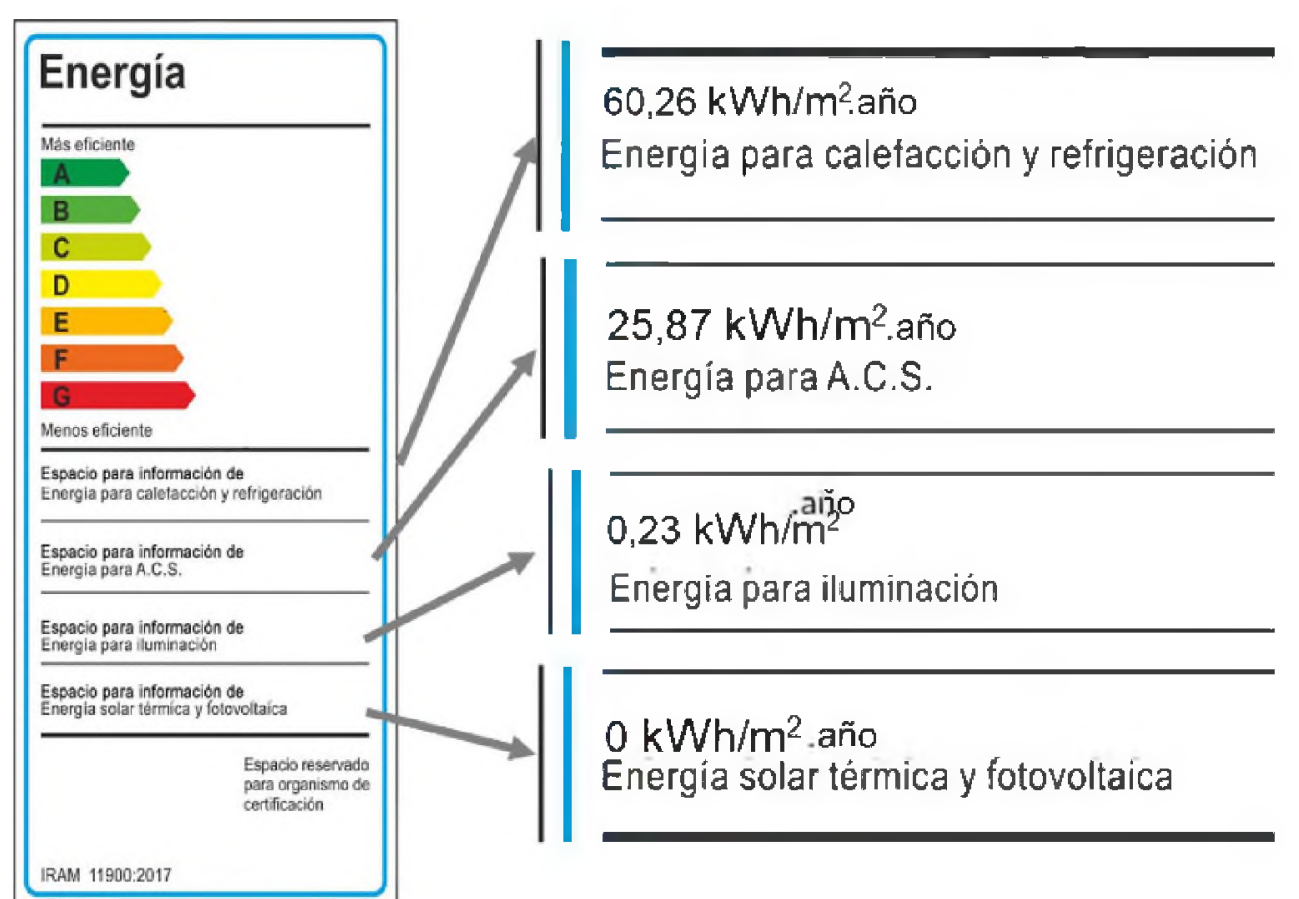

Figura 6 - Resultados según norma IRAM 
De la aplicación de la normativa fran- luador. Las recomendaciones se incluyen cesa, se obtienen los resultados de la tabla 7 en la evaluación. En la tabla 9 se represencon las respectivas etiquetas. En la Tabla 8 tan los gastos asociados al consumo enerse aprecian los valores que se obtendrían si gético con y sin aplicación de las recomense aplicasen las recomendaciones del eva- daciones.

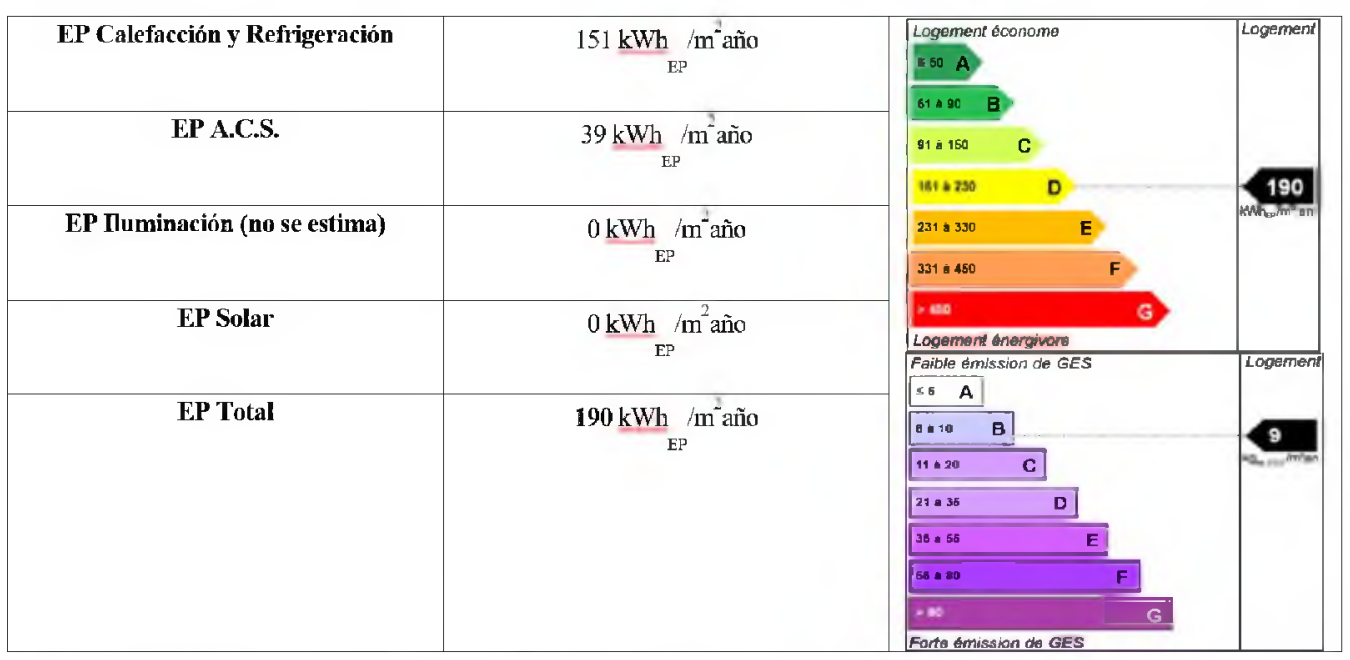

Tabla 8- Resultados aplicación normativa francesa

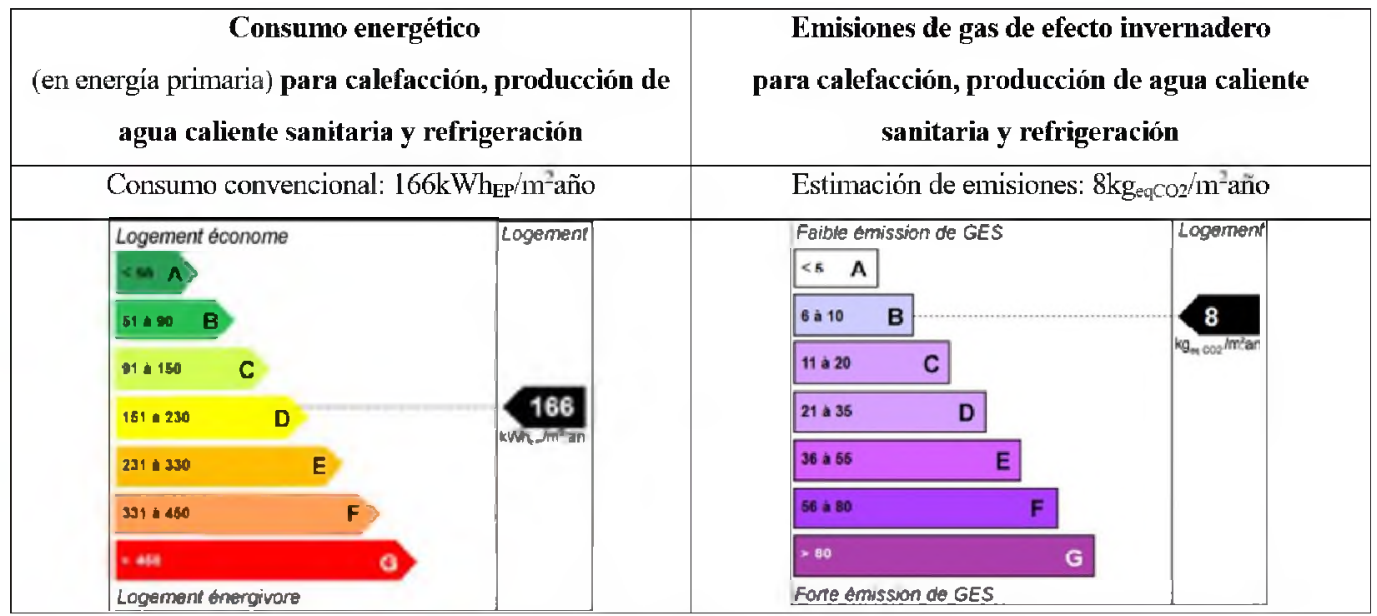

Tabla 9- Etiquetas con las aplicaciones de las recomendaciones

\begin{tabular}{|l|l|}
\hline Gastos anuales según el estado actual & Gastos anuales con aplicación de recomendaciones \\
\hline $2037 €$ & $1777 €$ \\
\hline
\end{tabular}

Tabla 10

\begin{tabular}{|l|l|}
\hline Norma RAM 11900:2017 (ARG) & Diagnóstico de Eficiencia energética (FRA) \\
\hline $86,36 \mathrm{kWh} / \mathrm{m}^{2}{ }^{2}$ año & $190 \mathrm{kWh} \mathrm{kW}_{\mathrm{FP}}^{2}{ }^{2}$ año \\
\hline
\end{tabular}

Tabla 11 - Comparación de resultados entre las dos normativas 


\section{CONCLUSIONES}

Dado que el valor energético calculado por la normativa argentina es alrededor de dos veces menor que la norma francesa, la normativa argentina es más permisiva que ésta e implica que no hay una correlación entre los métodos de cálculo.

Por otro lado, la norma argentina no agrupa ni clasifica en una escala de eficiencia con letras, es decir, presenta los valores de forma separada. Tampoco, se pueden relacionar las normas para crear una base de datos de etiquetado de eficiencia energética.

La normativa argentina no muestra el ahorro energético que se produce al realizar mejoras en cuanto los parámetros bioclimáticos del edificio. Por esto, tampoco se pueden crear programas de ayuda económica a la mejora de las prestaciones energéticas.

No se puede asociar una escala de emisión de dióxido de carbono dada la inexistencia de correlación entre las metodologías. Entonces, no se puede estimar la mitigación de $\mathrm{CO} 2$ de tipo residencial, en cada vivienda. Esto, a su vez, impide la aplicación de medidas para la reducción de emisiones de GEI producto del consumo energético residencial, el cual, a su vez, es el mayor consumidor de energía.

Adicionalmente, la diferencia entre resultados, llevarían a una incorrecta recomendación por parte del profesional, dado que al ser la normativa argentina más permisiva, no se tomarían los recaudos suficientes para realizar una mejora en cuanto a la eficiencia energética de una vivienda.
Además, las políticas desarrolladas a partir de la necesidad de mejora, no serían las suficientes si se aplicase la normativa argentina para la evaluación de la eficiencia energética.

Por lo expuesto, es necesaria una revisión y adaptación de la norma argentina, para que el etiquetado de eficiencia energética en un edificio sea correcto y para que las correspondientes decisiones políticas tengan coherencia con los lineamientos internacionales.

\section{BIBLIOGRAFÍA}

IRAM 11900:2017 - Prestaciones energéticas en viviendas. Método de cálculo.

Arrêté du 15 septembre (2006) - Relatif au diagnostic de performance énergétique pour les bâtiments existants proposés à la vente en France métropolitaine.

Arrêté du 17 octobre (2012) - Modifiant la méthode de calcul 3CL-DPE introduite par l'arrêté du 9 novembre 2006 portant approbation de diverses méthodes de calcul pour le diagnostic de performance énergétique en France métropolitaine.

La situación del mundo 1998: informe anual del Worldwatch Institute sobre medio ambiente y desarrollo (1998) - Lester R. Brown, Christoper Flavin y Hilary F. French ISBN 84742635149788474263510

DIRECTIVA 2002/91/CE DEL PARLAMENTO EUROPEO Y DEL CONSEJO de 16 de diciembre de 2002 relativa a la eficiencia energética de los edificios - Diario Oficial de las Comunidades Europeas

IRAM: ¿Cómo leer una etiqueta de eficiencia 
energética? http://www.eficienciaenergetica.org.ar/etiqueta. asp?id=eti

Alan S. Blinder, Economic Policy and the Great Stagflation (New York: Academic Press, 1979); Otto Eckstein, The Great Recession (Amsterdam: North-Holland, 1979); Mark E. Rupert and David P. Rapkin, "The Erosion of U.S. Leadership Capabilities, "in Paul M. Johnson and William R. Thompson, eds., Rhythms in Politics and Economics (New York: Praeger, 1985)

Philippe Collet - La réglementation thermique de 1974 à aujourd'bui - La RT 2012 bientôt en ordre de marche - Actu-Environnement bttps://www.actu-environnement.com/ae/ dossiers/energie-batiment-rt2012/historiquereglementation-thermique.php4 - Publicado el 20/06/2011

RT 2020 : tout sur la réglementation thermique 2020 - https://www.journaldunet.fr/patrimoinelguide-de-l-immobilier/1202193-rt2020-tout-sur-la-reglementation-thermique-2020/ - Mis à jour le 18/10/18 09:00

Ministerio de ambiente y desarrollo sustentable de la República Argetina (2017) - Segundo informe bienal de actualización de la república argentina a la convención marco de las naciones unidas sobre el cambio climático.

Hsu, D., How much information disclosure of building energy performance is necessary? Energy Policy, 2014. 64: p. 263-272.

Lu, T., X. Lü and M. Viljanen (2015) - ANew Method for Modeling Energy Performance in Buildings - Energy Procedia. 75: p. 18251831.

DIRECTIVA 2010/30/UE DEL PARLAMENTO EUROPEO Y DEL CONSEJO (2010) - Relativa a la indicación del consumo de energía y otros recursos por parte de los productos relacionados con la energía, mediante el etiquetado y una información normalizada.

Ministerio de la transición ecológica y solidaria: Ministère de la transition écologique et solidaire: ttps://www.ecologique-solidaire.gouv. $f r /$ diagnostic-performance-energetique-dpe Actualizado el 23 de mayo de 2018.

Ministère de l'Emploi, de la Cohésion Sociale et du Logement, Arrêté du 6 mai 2008 portant confirmation de l'approbation de diverses méthodes de calcul pour le diagnostic de performance énergétique en France métropolitaine

Agence Nationale de l'Habitat (ANAH) - Comunicado de prensa "Les clés de l'évaluation énergétique" - 3 de noviembre de 2014. 